\title{
Use of a Novel Bicarbonate-Based Impella 5.5 Purge Solution in a Coagulopathic Patient
}

\author{
Kyle Simonsen ${ }^{1}$, Brady Gunn ${ }^{2}$, Amber Malhotra ${ }^{3}$, Daniel Beckles ${ }^{1}$, Michael Koerner ${ }^{4}$, \\ Giuseppe Tavilla ${ }^{1}$, and Ramachandra Reddy ${ }^{3}$ \\ ${ }^{1}$ Baylor Scott \& White Medical Center Temple \\ ${ }^{2}$ A T Still University \\ ${ }^{3}$ Scott and White Memorial Hospital \\ ${ }^{4}$ Affiliation not available
}

September 25, 2021

\begin{abstract}
The Impella 5.5 with SmartAssist (Abiomed; Danvers, MA) is a life-saving treatment option in acute heart failure which utilizes a continuous heparin purge solution to prevent thrombosis. In patients with contraindications to heparin, alternative anticoagulation strategies are required. We describe the stepwise management of anticoagulation in a coagulopathic patient with persistent cardiogenic shock following a coronary artery bypass procedure who underwent Impella 5.5 placement. A direct thrombin inhibitor-based purge solution was utilized while evaluating for heparin-induced thrombocytopenia. Use of a novel bicarbonate-based purge solution (BBPS) was successfully used due to severe coagulopathy. There were no episodes of pump thrombosis or episodes of severe bleeding on the BBPS and systemic effects of alkalosis and hypernatremia were minimal.
\end{abstract}

\section{Hosted file}

BBPS cover letter.pdf available at https://authorea.com/users/436259/articles/538709-use-ofa-novel-bicarbonate-based-impella-5-5-purge-solution-in-a-coagulopathic-patient

\section{Hosted file}

BBPS case report.pdf available at https://authorea.com/users/436259/articles/538709-use-ofa-novel-bicarbonate-based-impella-5-5-purge-solution-in-a-coagulopathic-patient 